\title{
Design of Voltage control Oscillator using Nonlinear Composite Right/Left-Handed Transmission Line
}

\author{
Hala J. El-Khozondar ${ }^{1}$, Mahmoud Abu-Marasa ${ }^{1}$, Rifa J. El-Khozondar ${ }^{2}$, Mady Elbahri ${ }^{3}$, Said \\ Zouhdi $^{4}$ \\ ${ }^{1}$ Electrical Engineering Department, Islamic University of Gaza, Gaza, Palestine; Email:hkhozondar@iugaza.edu; \\ mah_marasa@hotmail.com \\ ${ }^{2}$ Physics Department, Al-Aqsa University, Gaza, Palestine; Email:rifa20012002@yahoo.com \\ ${ }^{3}$ Nanochemistry and nanoengineering, Faculty of Engineering, University of Kiel, Kiel, Germany; Email:me@ me@tf.uni- \\ kiel.de \\ ${ }^{4}$ Laboratorie do Génie Electrique de Paris, Université Paris Sud 11, France; Email: said.zouhdi@supelec.fr \\ *corresponding author, E-mail: hkhozondar@iugaza.edu
}

\begin{abstract}
In the present work, we propose a voltage control oscillator (VCO) at high frequency consists of nonlinear composite right/left-handed transmission line (CRLH-TL) loaded with Resonant Tunneling Diode (RTD). We designed three prototype device examples. The first one consists of one cell with short circuit at the beginning of the cell between ground and patch, and $50 \Omega$ load resistance were added at the end of the cell between ground and patch. The second one is similar to the first prototype but with open circuit at the beginning of the cell instated of short circuit. The third prototype consists of one cell with two $50 \Omega$ load resistances added between ground and patch at the beginning and at the end of the cell. The proposed VCO models are capable of generating oscillations at frequencies between 4.87- $14.9 \mathrm{GHz}$.

In our simulations, we used OrCAD and ADS software to analyze the proposed circuit.
\end{abstract}

\section{Introduction}

Voltage controlled oscillator (VCO) has several applications in communications and sensing network. It plays an important role in converting DC values that detected from thermometer, $\mathrm{pH}$ sensor, or pressure sensor into frequencies. The focus of this work is on designing VCO using artificial arrangement known as CRLH materials (CRLHM) and RTD. Left handed materials (LHM) are artificial materials which have both permittivity ( $\varepsilon)$ and permeability $(\mu)$ negative. The theoretical investigation of these materials was laid by Veselago [1]. The possibility of experimentally fabricating LHM [2] opened the door to design novel types of devices; $i$. $e$., perfect lens [3], amplification [4], sensor [5-6], and isolator [7-8]. Right handed materials (RHM) are 1 materials process positive refractive index.

Complementary resonant frequency of split-rings resonators (CSRRs) is responsible for giving negative effective permittivity to metamaterials. [9]. The first left-handed transmission line based on CSRRs was implemented by etching series capacitive gaps in the conductor strip, above the positions occupied by the CSRRs [10] where the series capacitive gaps are responsible for negative effective permeability. Thus, by combining these elements (gaps and CSRRs) at the resonance frequency of the resonators a lefthanded behavior is achieved in that band.

RTD has interesting applications referring to its high switching speed where it can reach up to $2.2 \mathrm{THz}$ and to its I-V characteristic that often exhibits negative differential resistance regions [11-12]. The RTD is placed parallel with shunt inductor of CRLH-TL hybrid approach as shown in Fig. 1. The hybrid resonant unit cell consists of a microstrip line with a series gap and two shunt stubs etched in the strip and a complementary split-rings resonators (CSRR) are etched in the ground plane (in grey) [13]. Metallic parts are depicted in black for the top layer and in grey for the bottom layer for substrate.

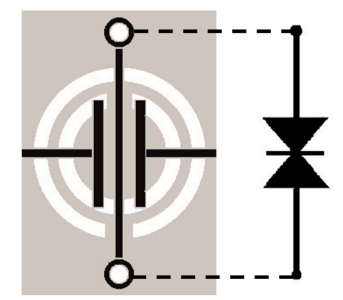

Figure 1: RTD at shunt with grounded stubs of the hybrid left-handed cells.

Next section presents the characteristics of the RTD. Section 3 introduces the equivalent circuit of the proposed system with detailed result analysis, followed by conclusion.

\section{Resonant Tunneling Diode (RTD)}

RTD has unique applications due to the high switching speed and to its negative differential resistance (NDR). The RTD I-V characteristic curve is displayed in Fig. 2. The 
curve exhibits the nonlinear behavior of the RTD diode. The circled region indicates to the NDR region, while the regions below and above it have positive resistance values. The characteristic curve shown in Fig. 2 is formulated by the following expression [14].

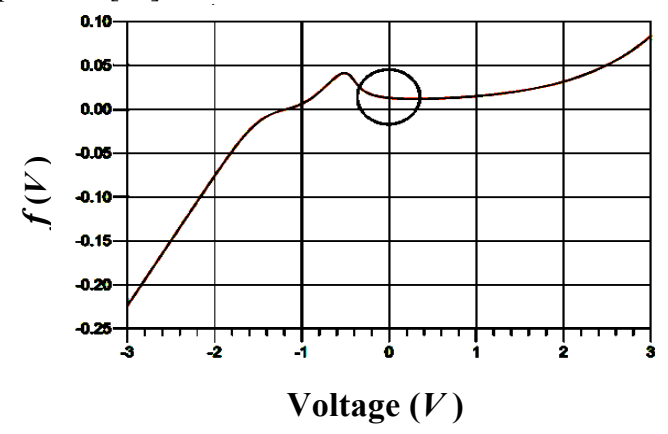

Figure 2: I-V characteristics of RTD. The circle marks the NDR region.

$$
f(V)=A \ln (\alpha)+H\left(e^{n_{2} q V(t) / K_{B} T}-1\right)
$$

where

$$
\alpha=\left[\frac{1+e^{q\left(B-C+n_{1} V(t)\right) / K_{B} T}}{1+e^{q\left(B-C-n_{1} V(t)\right) / K_{B} T}}\right]\left[\frac{\pi}{2}+\tan ^{-1}\left(\frac{C-n_{1} V(t)}{D}\right)\right],
$$

is the current density as function of $V$ which is the voltage cross RTD, $K_{B}, T$, and $q$ are Boltzmann constant, temperature in Kelvin and electron charge in coulombs, respectively. $A, B, C$ and $D$ are the dependent parameters on the device's physics. The experimental RTD I-V characteristic is fitted using the physics based description of the RTD equation in Fig. 2. The fitting parameters are taken from [15] as follows, $A=6.48 \times 10^{-3}, B=0.0875, C=$ $0.1449, D=0.02132, H=7.901 \times 10^{-4}, n_{1}=0.1902, n_{2}=$ 0.0284 , and $T=300 \mathrm{~K}$.

\section{Equivalent circuit, Results and analysis}

Fig. 3 is the equivalent circuit model of one cell loaded with RTD that introduced in Fig. 1.

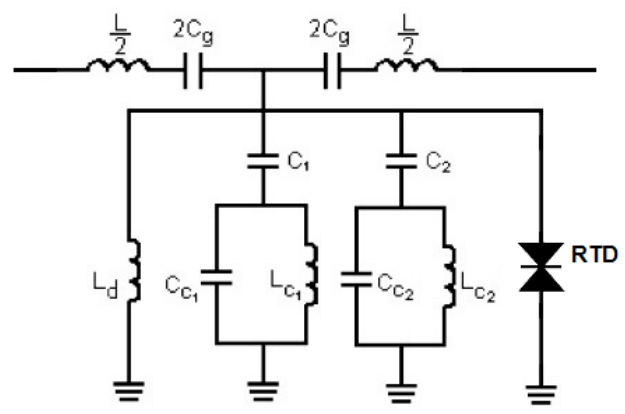

Figure 3: Equivalent circuit model of the unit cell (Fig. 1).

We designed three prototype device examples to achieve the desired design. The first one is a single cell with short circuit at the beginning of the cell and $50 \Omega$ load resistance at the end of the cell. The second one is similar to the first prototype but with open circuit at the beginning of the cell instated of short circuit. The third prototype consists of one cell with two $50 \Omega$ resistances at both the beginning and at the end of the cell. The equivalent circuit model of the three prototypes are shown in Fig. 4 (a, b, c) respectively. The RTD was biased using a variable DC voltage supply producing voltage oscillator at resistance load when the RTD biased in the NDR region. The DC voltage source was put at series with RTD to shift the I_V characteristic to the NDR region.

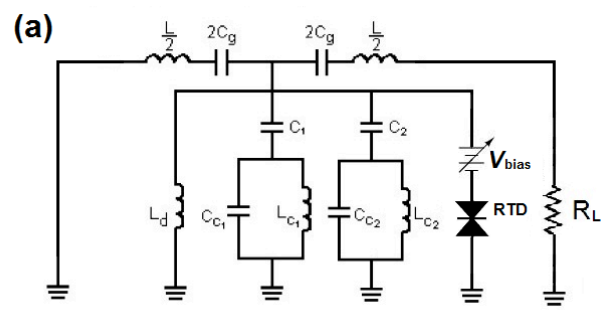

(b)

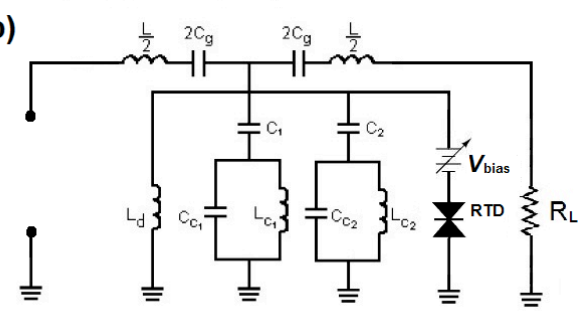

(c)

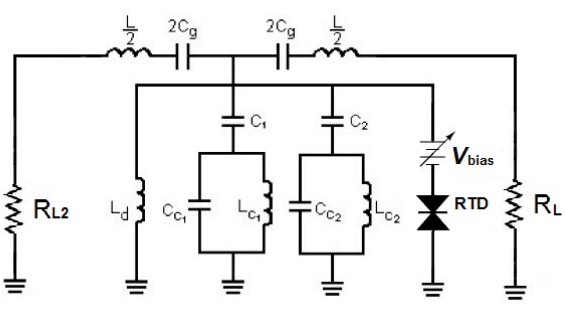

Figure 4: The equivalent circuit model of the unit cell loaded with RTD. (a) Short circuit at the beginning of the cell while $50 \Omega$ resistance is loaded at the end of the cell. (b) Same as (a) but with open circuit at the beginning of the cell instead of short circuit. (c) Two $50 \Omega$ load resistances at the beginning and at the end of the cell.

Figs. 5, 6 and 7 present the simulation output voltage measured at $R_{L}$ in time and frequency domain for the three models respectively. The simulation has been employed at different values of $\mathrm{V}_{\text {bias. }}$. The output voltage in time domain for the first model when $V_{\text {bias }}$ equals $0.7 \mathrm{~V}$ is displayed in Fig. 5 (a) and its spectral frequency is exhibited in Fig. 5(b). While, Fig. 5 (c) and Fig. 5 (d) show the output voltage for the same model when $\mathrm{V}_{\text {bias }}$ equals $1.1 \mathrm{~V}$ in time domain and frequency domain respectively. From the frequency analysis, we noticed that VCO can operate at three frequencies which are 4.8, 9.7 and $14.6 \mathrm{GHz}$ when $\mathrm{V}_{\text {bias }}$ equals $0.7 \mathrm{~V}$ and operates only at $14.6 \mathrm{GHz}$ when $\mathrm{V}_{\text {bias }}$ equals $1.1 \mathrm{~V}$.

Fig. 6(a) and Fig. 6(b) display the output voltage in time domain around $R_{L}$ at value of $\mathrm{V}_{\text {bias }}$ equal to $0.8 \mathrm{~V}$ and its spectral frequency respectively. Fig. 6(c) and Fig. 6(d) present the output voltage in time and frequency domain when $\mathrm{V}_{\text {bias }}$ equals $1.1 \mathrm{~V}$. From the spectral frequency, we realize that VCO operates at two main frequencies 7.1 and 
14.4 $\mathrm{GHz}$ when $\mathrm{V}_{\text {bias }}$ equals $0.8 \mathrm{~V}$ and operates at $14.4 \mathrm{GHz}$ only when $\mathrm{V}_{\text {bias }}$ equals $1.1 \mathrm{~V}$.

Fig. 7(a) and Fig. 7(b) demonstrate the output voltage in time domain and frequency domain respectively when $\mathrm{V}_{\text {bias }}$ equals $0.9 \mathrm{~V}$. The output voltage measured in time domain and frequency domain are exhibited in Fig 7(c) and Fig. 7(d) respectively when $V_{\text {bias }}$ equals $1.1 \mathrm{~V}$. From the frequency spectral we conclude that VCO operates at main frequency equals $14.4 \mathrm{GHz}$ for either $\mathrm{V}_{\text {bias }}$ equals $0.9 \mathrm{~V}$ or equals $1.1 \mathrm{~V}$. Table 1 shows the output voltage at RL for the three models as a form of "cos" function to show the gain of any frequency relative to the other values. Where the term
"Noise" is referring to the generation of noise in the designated case. We noticed that the oscillation frequencies depends on the value of Vbias. From Table 1, we noticed that there are three operating frequencies 4.8, 9.7 and 14.6 $\mathrm{GHz}$ for the first model and these frequencies may change slightly according to the value of Vbias. There are two main frequencies around 7.2 and $14.3 \mathrm{GHz}$ for the second model and these frequencies may vary according to the value of Vbias. For the third model, there is only one operating frequency that does not change as the value of Vbias changes.

(a)

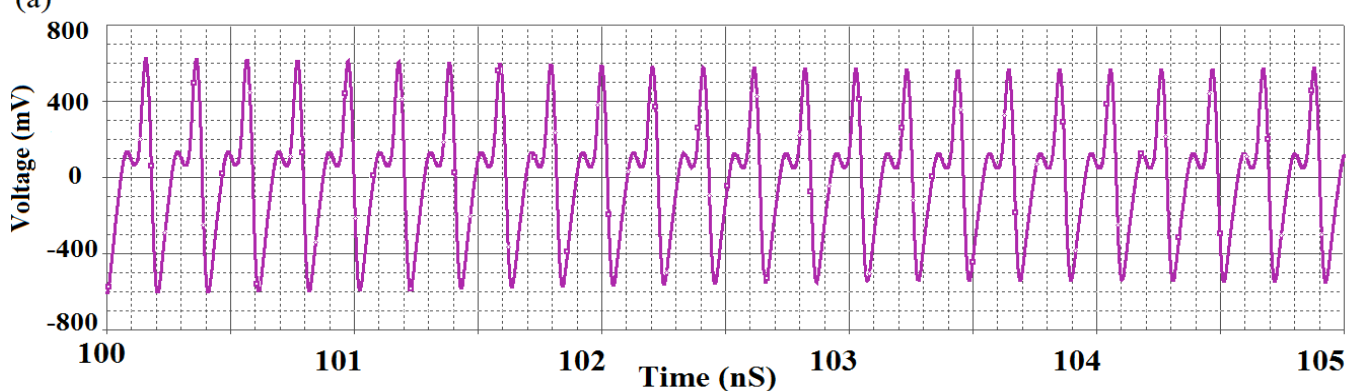

(b)
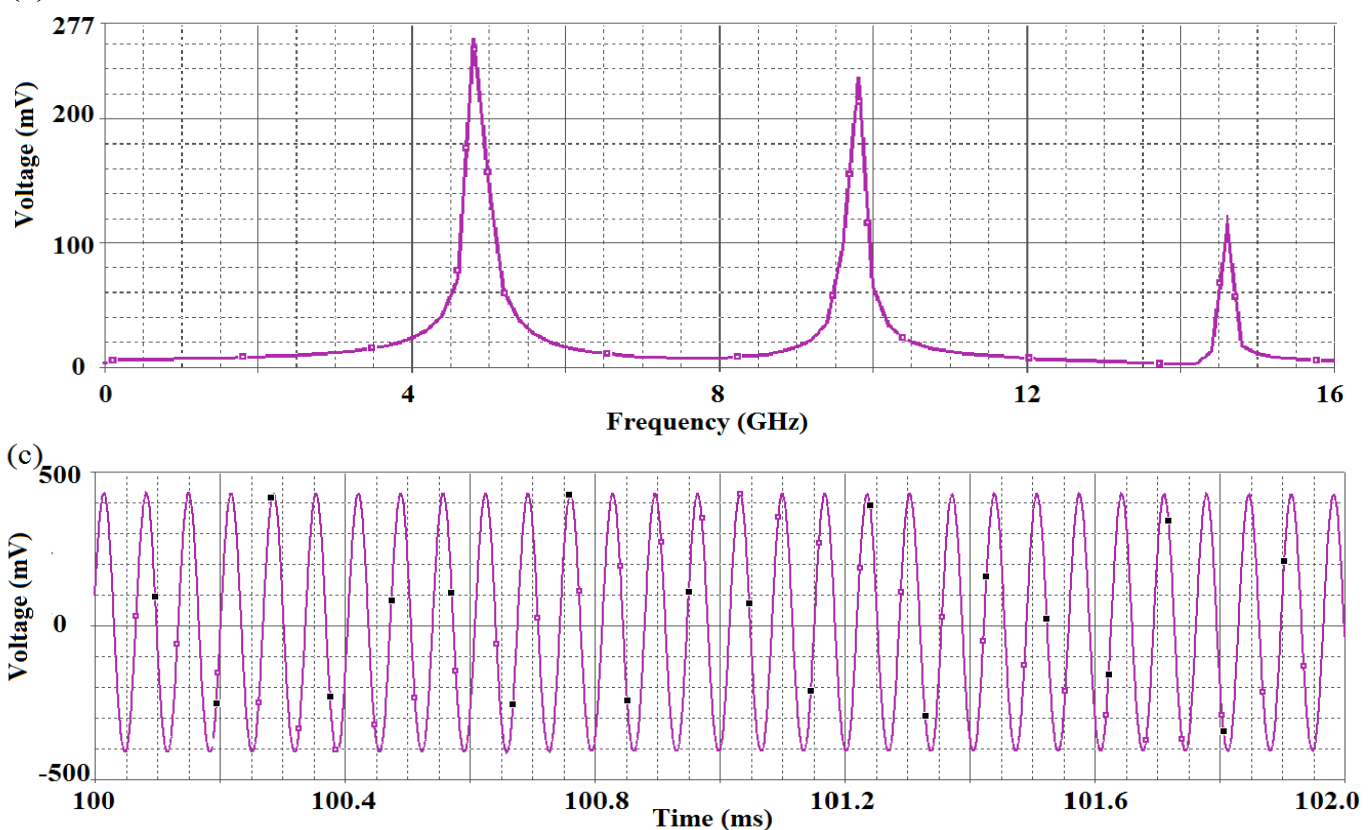

(d)

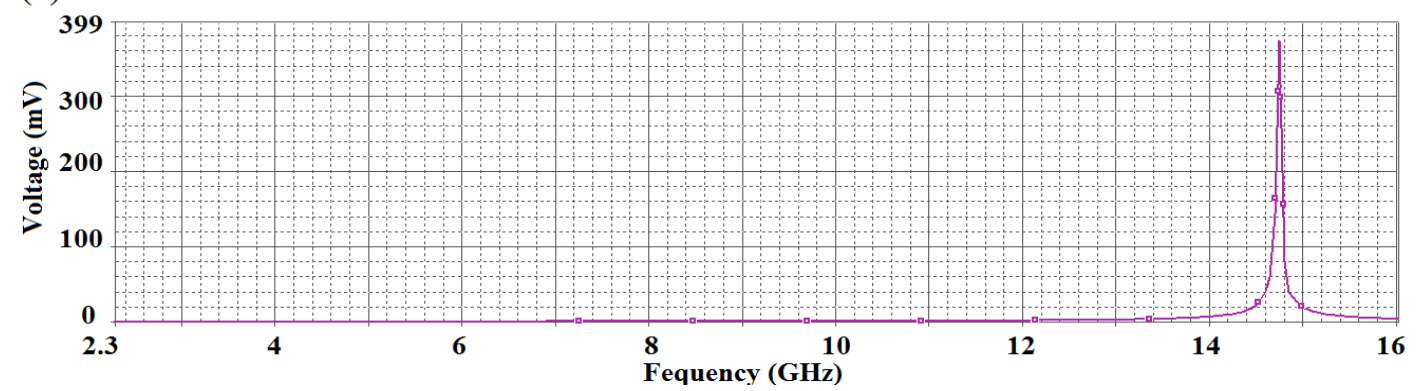

Figure 5: The simulation output voltage in time (a, c) and frequency domain (b, d) around $R_{L}$ for the first model (Fig. $4(\mathrm{a}))$ at two different values of $\mathrm{V}_{\text {bias. }}$ : $(\mathrm{a}, \mathrm{b})$ when $\mathrm{V}_{\text {bias }}$ equals $0.7 \mathrm{~V}$ while $(\mathrm{c}, \mathrm{d})$ when $\mathrm{V}_{\text {bias }}$ equals $1.1 \mathrm{~V}$. 

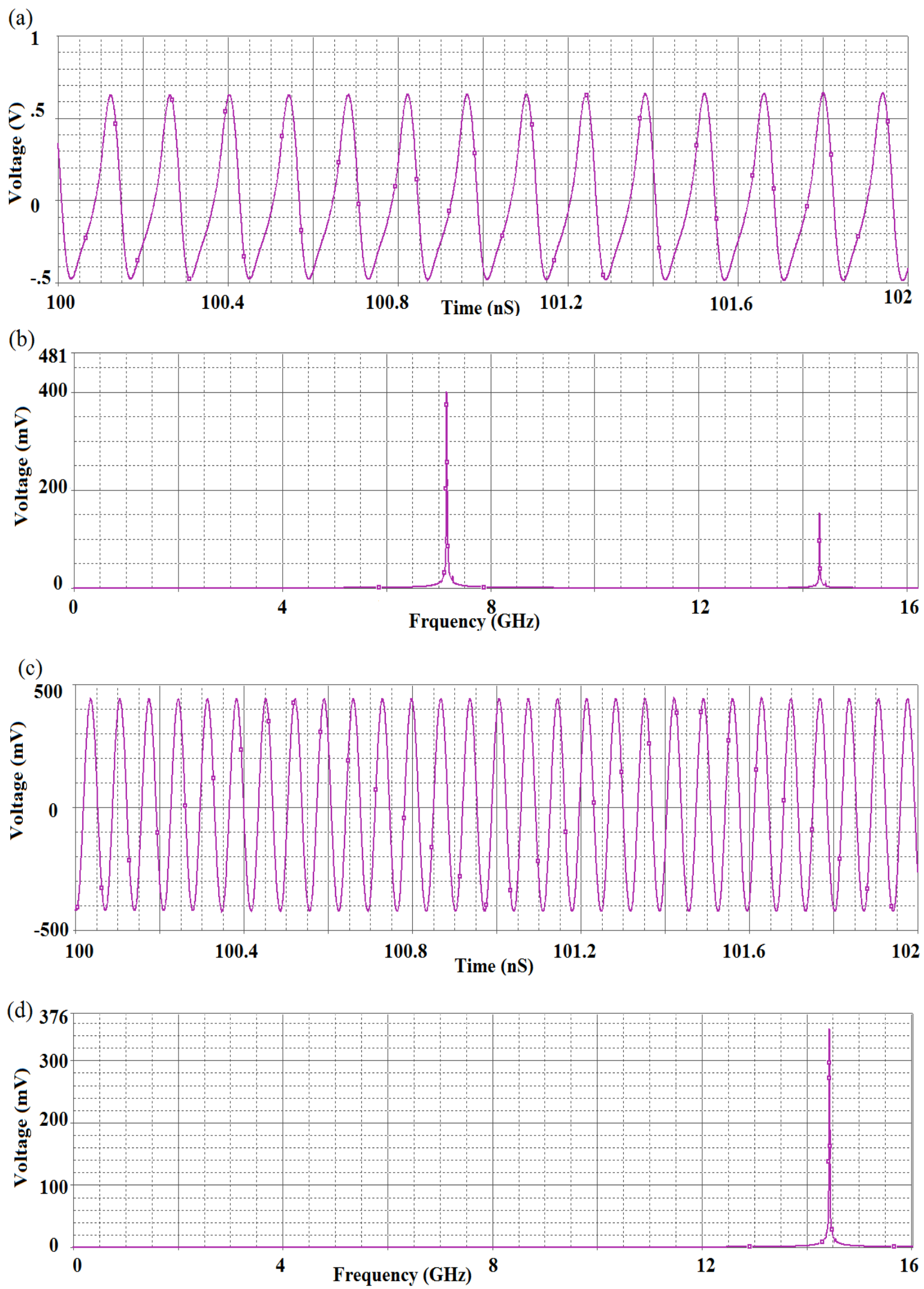

Figure 6: The simulation output voltage in time $(\mathrm{a}, \mathrm{c})$ and frequency domain $(\mathrm{b}, \mathrm{d})$ around $R_{L}$ for the second model (Fig. 4(b)) at two different values of $\mathrm{V}_{\text {bias. }}$ : $(\mathrm{a}, \mathrm{b})$ when $\mathrm{V}_{\text {bias }}$ equals $0.8 \mathrm{~V}$ while $(\mathrm{c}, \mathrm{d})$ when $\mathrm{V}_{\text {bias }}$ equals $1.1 \mathrm{~V}$. 

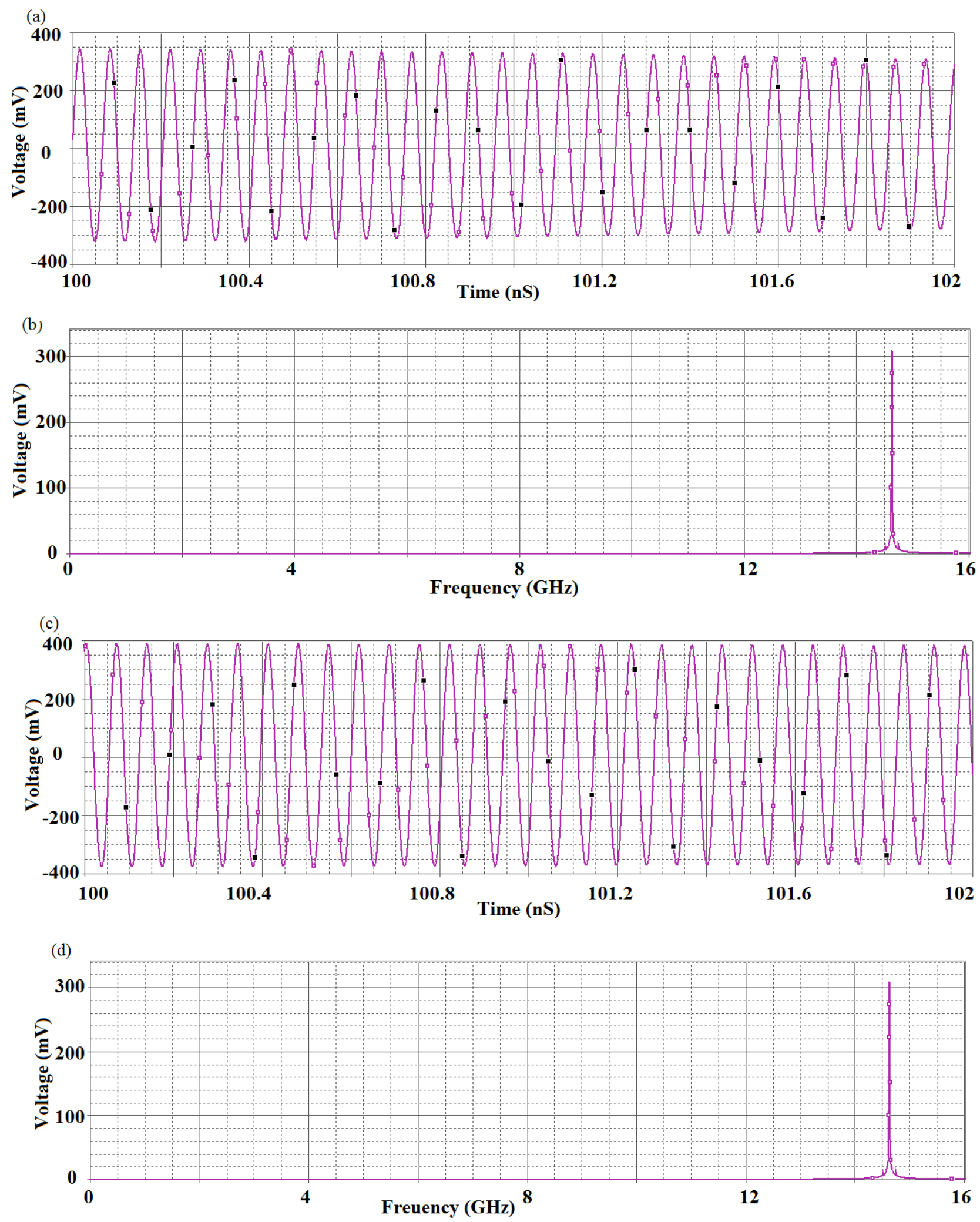

Figure 7: The simulation output voltage in time $(\mathrm{a}, \mathrm{c})$ and frequency domain $(\mathrm{b}, \mathrm{d})$ around $R_{L}$ for the third model (Fig. 4(c)) at two different values of $\mathrm{V}_{\text {bias. }}$ : $(\mathrm{a}, \mathrm{b})$ when $\mathrm{V}_{\text {bias }}$ equals $0.9 \mathrm{~V}$ while $(\mathrm{c}, \mathrm{d})$ when $\mathrm{V}_{\text {bias }}$ equals $1.1 \mathrm{~V}$. 
Table 1: Output voltage at $R_{L}$ for the three models as a Cos function

\begin{tabular}{|c|c|c|c|}
\hline$V_{\text {bias }}$ & First model $(\mathrm{mV})$ & Second model $(\mathrm{mV})$ & Third model $(\mathrm{mV})$ \\
\hline 0.7 & $\begin{array}{l}293 \operatorname{Cos}(2 \pi 4.87 \mathrm{G} \mathrm{t})+ \\
169 \operatorname{Cos}(2 \pi 9.75 \mathrm{G} \mathrm{t})+ \\
8.7 \operatorname{Cos}(2 \pi 14.6 \mathrm{G} t)\end{array}$ & Noise & Noise \\
\hline 0.75 & $\begin{array}{l}389 \operatorname{Cos}(2 \pi 4.91 G \mathrm{t})+ \\
294 \operatorname{Cos}(2 \pi 9.83 \mathrm{G} \mathrm{t})+ \\
111 \operatorname{Cos}(2 \pi 14.7 \mathrm{G} \mathrm{t})\end{array}$ & $\begin{array}{l}427 \operatorname{Cos}(2 \pi 7.12 G t)+ \\
147 \operatorname{Cos}(2 \pi 14.2 G t)\end{array}$ & Noise \\
\hline 0.8 & $\begin{array}{l}48 \operatorname{Cos}(2 \pi 4.94 G \mathrm{t})+ \\
27 \operatorname{Cos}(2 \pi 9.89 \mathrm{G} \mathrm{t})+ \\
9.8 \operatorname{Cos}(2 \pi 14.8 \mathrm{G})\end{array}$ & $\begin{array}{l}462 \operatorname{Cos}(2 \pi 7.15 G \mathrm{t})+ \\
38 \operatorname{Cos}(2 \pi 14.3 \mathrm{Gt})\end{array}$ & Noise \\
\hline 0.85 & $\begin{array}{l}280 \operatorname{Cos}(2 \pi 4.87 G \mathrm{t})+ \\
270 \operatorname{Cos}(2 \pi 9.75 G \mathrm{t})+ \\
230 \operatorname{Cos}(2 \pi 14.6 \mathrm{G})\end{array}$ & $\begin{array}{l}560 \operatorname{Cos}(2 \pi 7.19 G \mathrm{t})+ \\
180 \operatorname{Cos}(2 \pi 14.3 G \mathrm{t})\end{array}$ & $200 \operatorname{Cos}(2 \pi 14.5 \mathrm{G} \mathrm{t})$ \\
\hline 0.9 & $\begin{array}{l}290 \operatorname{Cos}(2 \pi 4.89 G t)+ \\
275 \operatorname{Cos}(2 \pi 9.79 G t)+ \\
190 \operatorname{Cos}(2 \pi 14.6 G t)\end{array}$ & $\begin{array}{l}600 \operatorname{Cos}(2 \pi 7.22 \mathrm{G} \mathrm{t})+ \\
190 \operatorname{Cos}(2 \pi 14.4 \mathrm{G} \mathrm{t})\end{array}$ & $240 \operatorname{Cos}(2 \pi 14.5 \mathrm{G} \mathrm{t})$ \\
\hline 0.95 & $\begin{array}{l}245 \operatorname{Cos}(2 \pi 4.90 G \mathrm{t})+ \\
320 \operatorname{Cos}(2 \pi 9.82 G \mathrm{t})+ \\
252 \operatorname{Cos}(2 \pi 14.7 \mathrm{G})\end{array}$ & $\begin{array}{l}620 \operatorname{Cos}(2 \pi 7.25 G \mathrm{t})+ \\
160 \operatorname{Cos}(2 \pi 14.5 \mathrm{G} \mathrm{t})\end{array}$ & $260 \operatorname{Cos}(2 \pi 14.6 \mathrm{G} \mathrm{t})$ \\
\hline 0.1 & $\begin{array}{l}280 \operatorname{Cos}(2 \pi 4.92 G \mathrm{t})+ \\
285 \operatorname{Cos}(2 \pi 9.84 G \mathrm{t})+ \\
260 \operatorname{Cos}(2 \pi 14.7 \mathrm{G}) \\
\end{array}$ & $275 \operatorname{Cos}(2 \pi 14.4 \mathrm{G} \mathrm{T})$ & $300 \operatorname{Cos}(2 \pi 14.6 \mathrm{G} \mathrm{T})$ \\
\hline 1.05 & $\begin{array}{l}310 \operatorname{Cos}(2 \pi 4.93 G \mathrm{~T})+ \\
321 \operatorname{Cos}(2 \pi 9.87 \mathrm{G})+ \\
181 \operatorname{Cos}(2 \pi 14.8 \mathrm{G} \mathrm{t})\end{array}$ & $344 \operatorname{Cos}(2 \pi 14.4 \mathrm{G} \mathrm{t})$ & $320 \operatorname{Cos}(2 \pi 14.6 \mathrm{G} \mathrm{t})$ \\
\hline 1.1 & $333 \operatorname{Cos}(2 \pi 14.7 \mathrm{G} \mathrm{t})$ & $315 \operatorname{Cos}(2 \pi 14.4 \mathrm{G} \mathrm{t})$ & $250 \operatorname{Cos}(2 \pi 14.6 \mathrm{G} \mathrm{t})$ \\
\hline 1.15 & $340 \operatorname{Cos}(2 \pi 14.7 \mathrm{G} \mathrm{t})$ & $280 \operatorname{Cos}(2 \pi 14.4 \mathrm{G} \mathrm{t})$ & $125 \operatorname{Cos}(2 \pi 14.6 \mathrm{G} \mathrm{t})$ \\
\hline 1.2 & $270 \operatorname{Cos}(2 \pi 14.7 \mathrm{G} \mathrm{t})$ & $270 \operatorname{Cos}(2 \pi 14.4 \mathrm{G} \mathrm{t})$ & Noise \\
\hline
\end{tabular}

\section{Conclusion}

We design VCO by adding RTD to CRLH-TL hybrid model introduced in [10]. We used DC source to bias the RTD such that it operates at NDR region. Three prototype device examples are designed. It is found that the oscillation frequencies can vary according to the DC biasing. The VCO capable of generating oscillations at frequencies ranges between $4.87 \mathrm{GHz}-14.9 \mathrm{GHz}$.

\section{References}

[1] V. G. Veselago, The electrodynamics of substance with simultaneously negative values of $\varepsilon$ and $\mu$, Sov. Phy.Usp. 10: 509-514, 1968.

[2] R. A. Shelby, D. R. Smith, and S. Schultz, Experimental verification of a negative index of refraction, Science 292: 77-79, 2001.

[3] J. B. Pendry, Negative refraction makes a perfect lens, Phys. Rev Lett. 85: 3966-3969, 2000.

[4] A. Gribe and G. Eleftheriades, Growing evanescent waves in negative-refractive index, App. Phys. Lett. 82: 1815-1817, 2003.
[5] H. J. El-Khozondar, M. S. Müller, R. J. El-Khozondar, M. M. Shabat, A. W. Koch, Temperature sensitivity of TE double-negative metamaterial optical sensor, Poc. Of SPIE Vol. 7390: 73900A 1-8, 2009.

[6] H. J. El-Khozondar, M. Müller, R. J. El-Khozondar, M. M. Shabat, A. W. Koch, Sensitivity of double-negative metamaterial optical sensor, International Journal of Pure and Applied Sciences and Technology (IJPAST) 11: 29-39, 2012.

[7] H. J. El-Khozondar, R. J. El-Khozondar, M. M. Shabat, A. W. Koch, Coupling efficiency of nonreciprocal optical isolator with metamaterials substrate and linear cladding, Metamaterials: Fundamentals and Applications II, Conference 7392 - Proceedings of SPIE Volume 7392: 2 - 5, 2009.

[8] R. El-Khozondar, H. El-Khozondar, M. Shabat, Applications of metamaterials in optical waveguide isolator, Journal of Al-Aqsa University series of natural science 12: 35-50, 2008.

[9] J. D. Baena, J. Bonache, F. Martín, R. Marqués, F. Falcone, T. Lopetegi, M. A. G. Laso, J. García, I. Gil, and M. Sorolla, Equivalent circuit models for split ring resonators and complementary split rings resonators coupled to planar transmission lines, IEEE Trans. Microw. Theory Tech. 53: 1451-1461, 2005. 
[10] M. Gil, J. Bonache, J. Garcia-Garcia, J. Martel, and F. Martin, Composite right/left-handed metamaterial transmission lines based on complementary split-rings resonators and their applications to very wideband and compact filter design, IEEE Trans. Microw. Theory Tech. 55: 1296-1304, 2007.

[11] J. Ling, Resonant tunneling diodes: Theory of operation and applications, University of Rochester, Rochester, NY.

[12] M. G. Barba, Resonant-type metamaterial transmission lines and their application to microwave device design, Doctoral Thesis, Universitat Autònoma de Barcelona ,2009

[13] M. Gil, J. Bonache, J. Garcia-Garcia, J. Martel, and F. Martin, Composite right/left-handed metamaterial transmission lines based on complementary split-rings resonators and their applications to very wideband and compact filter, IEEE Trans. Microw. Theory Tech. 55: 1296-1304, 2007.

[14] M. Reddy, Schottky-collector resonant tunnel diodes for sub-millimeter-wave applications, University of California Santa Barbara, 1997.

[15] T. J. Slight, B. Romeira, L. Wang, J. M. L. Figueiredo, E. Wasige, \& C. N. Ironside, A Liénard Oscillator Resonant Tunnelling Diode-Laser Diode Hybrid Integrated Circuit: Model and Experiment, IEEE journal of quantum electronics 44: 1158-1163, 2008. 